\section{The sight of art}

\section{J.D. Mollon}

\section{Cameraworks.}

By David Hockney.

Thames \& Hudson/Knopf: 1984. Pp.280. £30, $\$ 50$.

ADVANCES in visual science have more than once prompted a fashion in art. Pointillism and Cubism offer two examples. But whatever the theoretical commentaries provided by the artist, the connection between the perceptual theory and the art is usually loose or incomplete, and the art finally stands or fails on its own merits.

With Cameraworks, his new collection of photographic collages, David Hockney has joined the tradition of artists who offer a scientific rationale for their work. He has seized on an aspect of visual perception that has exercised experimental psychologists during the past two decades but yet is little understood.

When we look at a visually busy scene, such as Epsom Downs on Derby Day, the Grand Canyon from the North Rim or the interior of King's Chapel, it seems as if we have an immediate and richly detailed grasp of a panorama that subtends at least 100 degrees at our eye. Yet, if we fixate our eyes steadily on one point, we discover that our detailed perceptual grasp is limited to about 1.5 degrees, which corresponds to the tiny foveolar region of our retina.

We normally do not constrain our eyes to look fixedly at one spot, and, almost without the awareness of their owner, they dance around the scene, dwelling on any given point for about 250 milliseconds. The two eyes are yoked in their movements; and their angle of convergence (together with their focal length) is automatically adjusted as we look from background to foreground. From a train of successive fixations, our brain builds up for us an apparently detailed representation of an extended scene. If the scene is changing whilst we look, then the temporal variation is incorporated into our four-dimensional internal icon. And, to use the modern jargon, we seem to enjoy pan and scroll facilities in all the four dimensions.

Hockney is explicitly aware of this aspect of visual perception. $\mathrm{He}$ writes, for example: "There are a hundred separate looks across time from which I synthesize my living impression of you". He goes on to observe that it is not possible to look at a photograph, even an erotic one, for more than 30 seconds. And he argues, with a logic I cannot fully follow, that this is because a photograph, unlike a painting, takes very little time in the making. Hockney's solution is to take a multitude of snapshots of a scene or event and then to mount them in a collage.

He has foregone one major part of the aesthetic control open to the traditional photographer, the control of development and printing. He either has used Polaroid snaps or else has sent rolls of exposed negative round to his local neighbourhood processors, "Benny's Speed Cleaning and One-hour Processing". (Benny's used to send standardized notices back with the prints, patiently explaining to Hockney what he had done wrong, "how I should try to center the camera on the subject, focus on the foreground, and so forth"'.)

Does it come off? I think one must consider separately those cubist-like collages where Hockney jumbles the component images and those montages where there is only minimal perturbation of the topography of a scene. In the former cases, where several snaps of a face or object, taken at successive moments, may abut and overlap each other, one's attention can be held for 30 seconds. But the reason, I believe, is the very opposite of the one advanced by Hockney. It is not that the collage expresses the normal experience of looking at a person or scene. Rather, the contradictions between local depth cues in one area and those in an adjacent area, the discontinuities of form, the variations of facial mood, combine to thwart and delay the perceptual mechanism that is struggling to construct a single, intcrnally-consistent representation of the scene. Much as when we look at a still life by Braque, our visual system tries out successive hypotheses and is never able to attain coherence.

One of the best pieces in Hockney's collection - a portrait of the artist's mother at Bolton Abbey on a wet November day - is only spoilt by the intrusiveness of the collage. From the glistening blades of grass in the foreground, past Mother in her blue-green oilskin, to the grey-green graves beyond, the picture is marvellously coherent in its atmosphere; but the eye is interrupted by misfitting or missing bits of collage - and by the unevenness of Benny's processing.

One of the notable successes of the collage technique is in conveying the impression of rapid human movement. This can be well seen in the example reproduced here, where Hockney's spinning skater recalls classical Indian representations of the goddess Shiva.

In the case of the topographically coherent montages (for example his panoramic views of the Grand Canyon), I don't understand why Hockney doesn't save up the dollars he spends at Benny's and buy himself one of the specialist, slowly rotating, panoramic cameras that have been available since Friedrich von Martens introduced his daguerréotype panoramique in 1845 . But a competent classical photographer, using a fixed lens, could often surpass Hockney's montages: there are single shots of the Grand Canyon by Andreas Feininger that capture more of the awesomeness and volume of the place than do Hockney's amateur montages of the same subject. And I could go on looking at, say, Feininger's "Ninth Avenue Elevated" (1940) for much more than 30 seconds - indeed for longer than it took me to flick through to the end of Hockney's Cameraworks.

J.D. Mollon is a Lecturer in Experimental Psychology at the University of Cambridge.

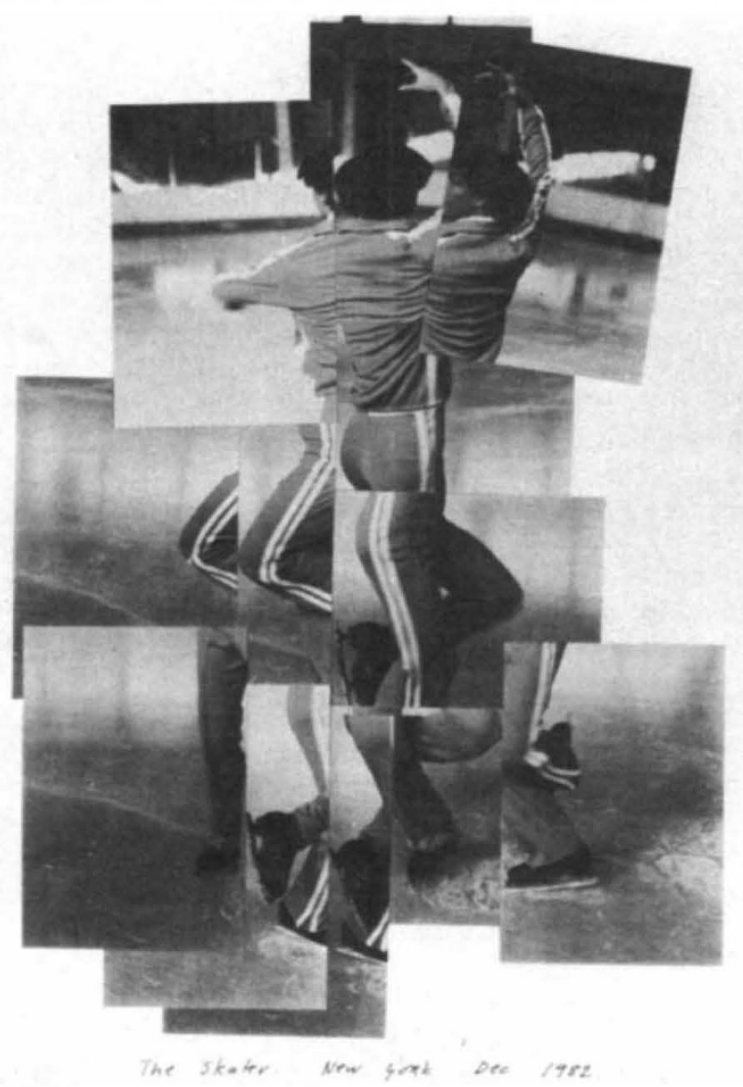

